INPLASY

PROTOCOL

To cite: Dambroz et al. The effect of physical fatigue on the performance of soccer players: a systematic review. Inplasy protocol 202150054. doi:

10.37766/inplasy2021.5.0054

\section{THE EFFECT OF PHYSICAL FATIGUE ON THE PERFORMANCE OF SOCCER PLAYERS: A SYSTEMATIC REVIEW}

Dambroz, F1; Clemente, FM2; Calvo, T3; Williams, M4; Teoldo, ${ }^{5}$.

Review question / Objective: What the effect of physical fatigue on the performance of soccer players?

Condition being studied: The condition studied was the physical fatigue of soccer players and its effects on players' performances.

Information sources: The information sources were the Web of Science (from 1945 to 2020), PubMed, and SPORTDiscus electronic databases. The reference lists of the selected articles were also analyzed.

INPLASY registration number: This protocol was registered with the International Platform of Registered Systematic Review and Meta-Analysis Protocols (INPLASY) on 15 May 2021 and was last updated on 15 May 2021 (registration number INPLASY202150054).

Review Stage at time of this

submission: Data analysis.

Conflicts of interest:

None declared.

\section{INTRODUCTION}

Review question / Objective: What the effect of physical fatigue on the performance of soccer players?

Condition being studied: The condition studied was the physical fatigue of soccer players and its effects on players' performances.

\section{METHODS}

Search strategy: Searches for scientific articles were performed in the following electronic databases: Web of Science (from 
1945 to 2020), PubMed, and SPORTDiscus. The terms used for the searches were: (soccer OR soccer) AND ("neuromuscular fatigue" OR "muscular fatigue" OR "physical fatigue" OR fatigue OR "physical exertion" OR "physical load") AND (performance).

Participant or population: Healthy soccer players from any age group, competitive level or sex.

Intervention: Exposure to physical fatigue.

Comparator: Pre and post-physical fatigue conditions.

Study designs to be included: No restrictions with regard to study design.

Eligibility criteria: i) articles published in international peer-reviewed journals; ii) validity and reliability of the instruments used in studies established and published in scientific journals; iii) research conducted with human beings; iv) articles related only to soccer; v) published in English, Portuguese or Spanish.

Information sources: The information sources were the Web of Science (from 1945 to 2020), PubMed, and SPORTDiscus electronic databases. The reference lists of the selected articles were also analyzed.

Main outcome(s): In this review the attention will be directed in order to understand each session of the selected articles from a look at the topics: studies focus; sample description; analysis of instruments and procedures; analysis of results; and guidance for future work.

Additional outcome(s): The articles identified in the present systematic review were classified as presenting high methodological quality $(\mathbf{7 7 . 8 0} \%)$. In general, the quality analysis suggested that the aims of the studies were clearly stated, the scientific literature of the area was revised, and the ethical procedures required for studies with human beings were respected. Also, the statistical procedures and the variables analyzed were well defined in the studies. The main methodological deficiencies identified by the quality assessment were the lack of justification for the sample size, the lack of information regarding voluntary dropouts, and the noninclusion or superficial presentation of the studies' practical implications. The results obtained in recent years in relation to the cognitive performance of soccer players in a state of physical fatigue have shown inconsistencies. On the other hand, studies have shown negative effects of physical fatigue on the players' physical and technical performance. In addition, there is a lack of studies addressing the individual and collective tactical performance of players in a state of physical fatigue.

Quality assessment / Risk of bias analysis: The studies' quality was assessed using a risk-of-bias quality form (16 items) adapted from Law and colleagues (1998).

Strategy of data synthesis: The initial screening was based on the analysis of the articles' title and summary. Duplicated articles between databases were excluded. Next, some articles were selected for fulltext analysis in order to verify if they meet all the inclusion criteria described by the PICOS strategy.

Subgroup analysis: Not applicable. A metaanalysis will not be performed.

Sensitivity analysis: Not applicable. A metaanalysis will not be performed.

Language: English.

Country(ies) involved: Brazil; Portugal; Spain; USA.

Keywords: Performance; fatigue; evaluation; soccer; decision making.

Contributions of each author:

Author 1 - Felipe Dambroz.

Email: felipe.dambroz@ufv.br

Author 2 - Filipe Clemente.

Author 3 - Tomás Calvo.

Author 4 - Mark Williams.

Author 5 - Israel Teoldo.

Email: israel.teoldo@ufv.br 